\title{
INTEREST IN PHYSICAL ACTIVITY DURING COVID-19 PANDEMIC AMONG COUNTRIES WITH DIFFERENT LOCKDOWN APPROACHES: A GOOGLE TRENDS ANALYSIS
}

\author{
Jobson Lopes de Oliveira ${ }^{1,2, \star}$, José de Paula Barbosa Neto², Francisco Airton Castro da Rocha \\ 1.Universidade Federal do Ceará, Fortaleza (CE), Brazil. 2.Centro Universitário Christus, Fortaleza (CE), Brazil. \\ *Corresponding author: jobson.oliveira88@gmail.com
}

\section{BACKGROUND}

The coronavirus disease 2019 (COVID-19) pandemic has led most countries to adopt strict isolation policies, such as homeconfinement for several months, forcing an abolition of regular outdoor exercise. However, some countries have chosen to take a different approach to cope with the outbreak without restricted lockdown measures. Inactivity and sedentary behavior have detrimental consequences to physical and mental health. Home based exercise programs are a feasible way to remain physically active during this period. The aim of this study is to investigate public interest in physical activity in countries that have adopted lockdown and countries that have not.

\section{MATERIALS AND METHODS}

Google Trends was used to obtain the daily number of searches for "physical activity" from September 2019 to August 2020 among countries that adopted strict lockdown measures (Brazil, Spain and Italy) and countries that opted for vertical isolation (Sweden and Turkey). A search was made using the term in the language of each country. All search query data are shown as proportionally popularity on a scale from 0 to 100 , with a value of 100 representing the peak popularity of a term in a selected time range and region.

\section{RESULTS}

In countries that adopted lockdown, the number of daily searches for "physical activity" has spiked since March 2020. However, although it still maintains high levels of search queries in Brazil until late August, by late May searches had meaningfully decreased in Spain and Italy, coinciding with relaxation of the social distancing measures (Fig. 1). On the other hand, Sweden and Turkey experienced a slight increase in the number of searches for physical activity in March, followed by a sharp drop until July 2020, which coincides with the reduction in the number of deaths in these countries (Fig. 2).

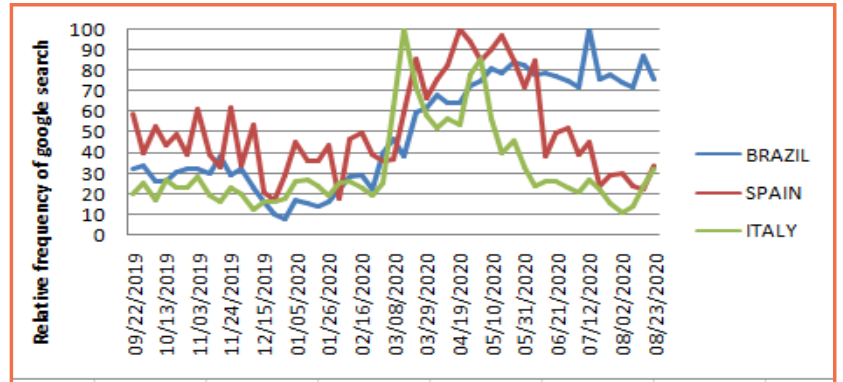

Figure 1. Google Trends for "physical activity" in countries taking a lockdown approach.

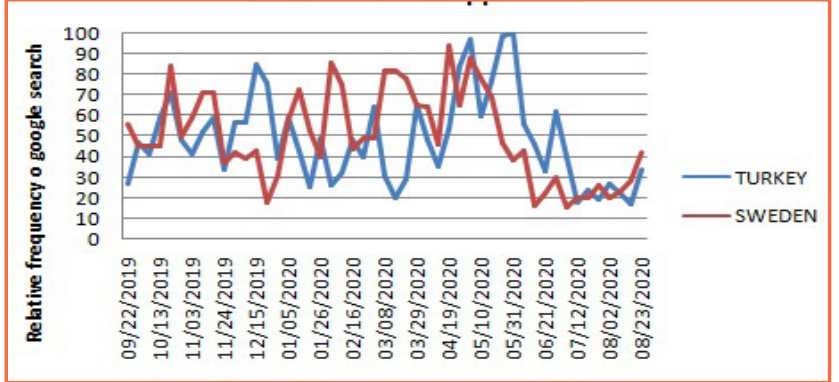

Figure 2. Google Trends for "physical activity" in countries taking a no-lockdown approach.

\section{CONCLUSION}

Public interest in physical activity, assessed through Internet search queries, has increased in countries that adopted lockdown during the COVID-19 pandemic. Regular exercises adapted to the conditions at home are important to address the detrimental consequences of sedentarism during the COVID-19 outbreak. Nevertheless, given the diversity of quality of information available on the Internet dedicated to the promotion of structured physical activity, it is important to consult reliable information created by health societies. 